\title{
BIAS CIRCUIT INSTABILITIES AND THEIR EFFECT ON THE d.c. CURRENT-VOLTAGE CHARACTERISTICS OF DOUBLE-BARRIER RESONANT TUNNELING DIODES
}

\author{
C. Kidner, I. Mehdi, J. R. East and G. I. Haddad \\ Center for High-Frequency Microelectronics and Center for Space Terahertz Technology, Department of \\ Electrical Engineering and Computer Science. The University of Michigan, Ann Arbor, MI 48109, U.S.A.
}

(Received 19 March 1990; in revised form 28 June 1990)

\begin{abstract}
Bias circuit stability has important implications for the study and application of double-barrier resonant tunneling structures. Stability criteria for resonant tunneling diodes are investigated for the common bias circuit topologies. A systematic study was made of the effect of different bias circuit elements on the measured d.c. I-V curves. A double-barrier diode was studied as an example, with experimental and theoretical results. The main results of the paper are (1) stable resonant tunneling diode operation is difficult to obtain, (2) the low-frequency oscillation introduces a characteristic signature in the measured d.c. $I-V$ characteristic
\end{abstract}

\section{NOTATION}

$C_{\mathrm{d}} \quad$ device capacitance

$C_{e}$ external capacitance

$G_{\mathrm{d}}$ device conductance

$i, J \quad$ electrical a.c. and d.c. current respectively

$I_{\text {plat }} \quad$ plateau current

$I_{\mathrm{p}}$ peak current

$I_{\mathrm{v}} \quad$ valley current

$L_{B} \quad$ inductance associated with the bias supply

$L_{\mathrm{s}} \quad$ series inductance

$R_{\mathrm{B}} \quad$ resistance associated with the bias supply

$\boldsymbol{R}_{\mathrm{d}} \quad$ device negative resistance

$R_{\mathrm{s}} \quad$ device series resistance

$V_{\mathrm{D}}$ voltage across the diode

$V_{\text {in }}$ voltage across the input terminals of the bias circuit

$V_{p} \quad$ voltage at the peak current

$V$. voltage at the valley current

$\AA$ angstroms

$\Omega$ ohms

$\omega$ frequency of oscillations in radians/s

$\omega_{r} \quad$ resistive cutoff frequency in radians/s

$\omega_{v}$ resonant frequency in radians $/ \mathrm{s}$

\section{INTRODUCTION}

Many of the proposed applications of double-barrier resonant tunneling structures make use of the structure's negative differential resistance (NDR). NDR is observed in d.c. current-voltage $(I-V)$ curves and has been shown to exist to at least $400 \mathrm{GHz}[1]$. However, not enough attention has been paid to the interaction of the resonant tunneling structure and the bias circuit used. This is particularly important when the device is biased in the NDR region. Improper choice of bias circuit elements can lead to a circuit which is not short-circuit stable. Instability can result in switching and bistable characteristics which mask the NDR region, or introduce low frequency oscillations in the bias circuit. For many applications, particularly highfrequency power generation, these oscillations render the device useless. The bias circuit element values published indicate that many workers have underestimated the severity of the problem. Also, the correlation among the different types of d.c. I-V curve anomalies and specific bias circuit elements has not been established by previous workers. Requiring stability also impacts applications of the Resonant Tunneling Diode (RTD). For instance, the stability requirements will reduce the power available from RTDs. This effect is discussed elsewhere[2].

This paper reports a systematic study of how the bias circuit affects the measured d.c. I-V curve of an RTD. The paper is organized as follows. In the next section the stability criterion for the typical bias circuit topology is presented. The effect of the bias circuit elements on stability is discussed. Using this criterion an RTD is stabilized in its NDR region. In Section 3 the bias circuit elements are varied to demonstrate theoretically and experimentally how the commonly observed d.c. $I-V$ curve anomalies are correlated to bias circuit elements.

\section{LOW FREQUENCY STABILITY ANALYSIS}

The RTD is a voltage controlled negative resistance device. This means that the device will be connected through a bias circuit to a voltage source. In a practical circuit the bias circuit will include the power supply source impedance and various parasitic elements. If the two terminal circuit consisting of the RTD and its bias circuit is not short circuit stable there will be unwanted oscillations in the bias circuit. A two terminal circuit is short circuit stable if there are no zeroes of the impedance for complex frequencies with positive real parts. The simplest bias circuit 
(a)

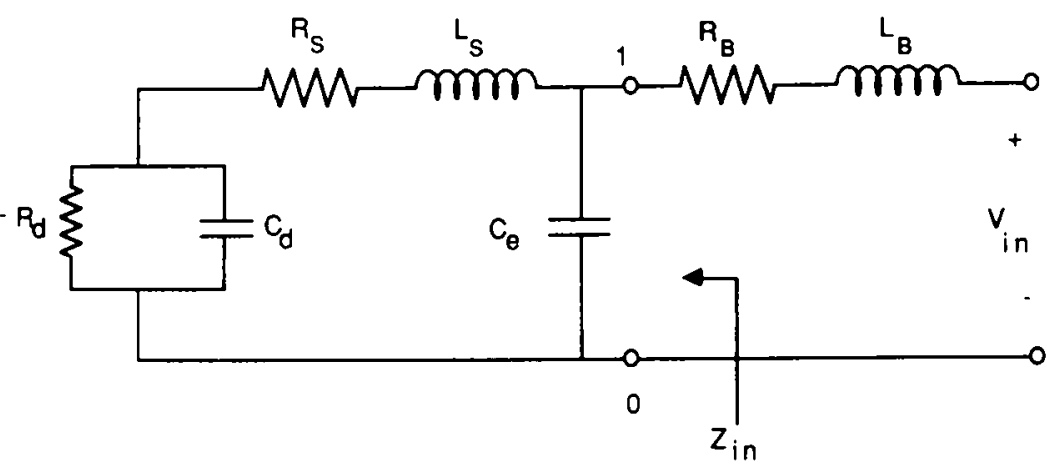

(b)

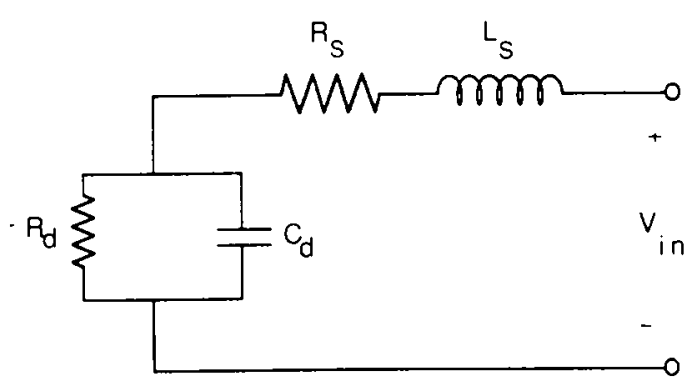

Fig. 1. (a) A generalized equivalent circuit for resonant tunneling diodes including the bias circuit and an external capacitance. (b) The simplified equivalent circuit used for stability analysis.

topology for the RTD is shown in Fig. 1(a). $-R_{\mathrm{d}}$ and $C_{\mathrm{d}}$ represent the NDR and capacitance of the double barrier structure, respectively. $R_{\mathrm{s}}$ is the positive parasitic resistance of the device plus any external resistance introduced by the bias circuit. $L_{\mathrm{s}}$ is the inductance introduced by the contact wire. $C_{c}$ is an external capacitance added for stability. $L_{\mathrm{B}}$ and $R_{\mathrm{B}}$ are the inductance and the resistance of the bias lines. This is the same equivalent circuit as was used for the tunnel diode. In the early 1960's many papers concerning tunnel-diode stability were published. While some applications called for short-circuit unstable devices[3], most used devices which were shortcircuit stable[3,4]. The stability criterion for the circuit was developed by Hines[5]. In the simplest case the stabilizing capacitor, $C_{\mathrm{e}}$ is not used. Letting $L_{\mathrm{s}}+L_{\mathrm{B}} \rightarrow L_{\mathrm{s}}$ and $R_{\mathrm{s}}+R_{\mathrm{B}} \rightarrow R_{\mathrm{s}}$ the circuit reduces to Fig. 1(b). By solving the associated differential equation it can be shown that the stability criterion is given by

$$
\frac{L_{\mathrm{s}}}{C_{\mathrm{d}} R_{\mathrm{d}}^{2}}<\frac{R_{\mathrm{s}}}{R_{\mathrm{d}}}<1 .
$$

Algebraic manipulation shows this is equivalent to requiring

$$
\omega_{\mathrm{r}}<\omega_{\mathrm{x}} .
$$

where the cutoff frequency of the diode

$$
\omega_{\mathrm{r}}=\frac{G_{\mathrm{d}}}{C_{\mathrm{d}}} \sqrt{\frac{1}{R_{\mathrm{s}} G_{\mathrm{d}}}-1}
$$

is the frequency at which the real part of the circuit impedance is zero and the resonant frequency

$$
\omega_{\mathrm{r}}=\sqrt{\frac{\mathrm{l}}{L_{\mathrm{s}} C_{\mathrm{d}}}-\frac{G_{\mathrm{d}}^{2}}{C_{\mathrm{d}}^{2}}}
$$

is the frequency at which the imaginary part is zero. The stability may also be described in terms of a stability diagram as shown in Fig. 2. The different regions of the diagram correspond to different types of solutions to the associated differential equation. In region I the solutions grow exponentially. In region II the solutions grow sinusoidally. In region III the solutions are damped sinusoids. In region IV the solutions decay

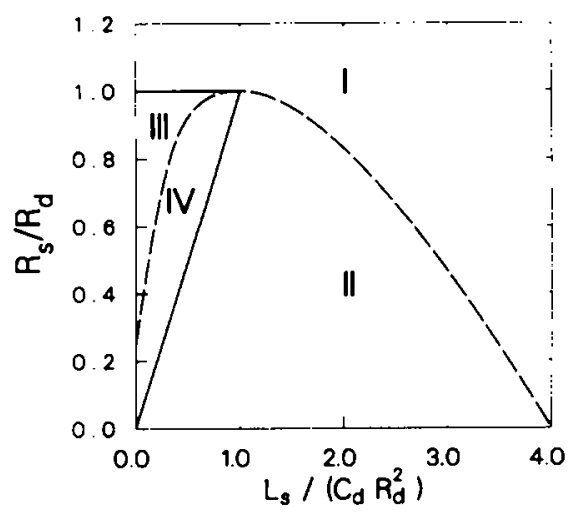

Fig. 2. The stability diagram for resonant tunneling diodes including circuit parameters. Regions III and IV correspond to a short circuit stable circuit. 
(a)

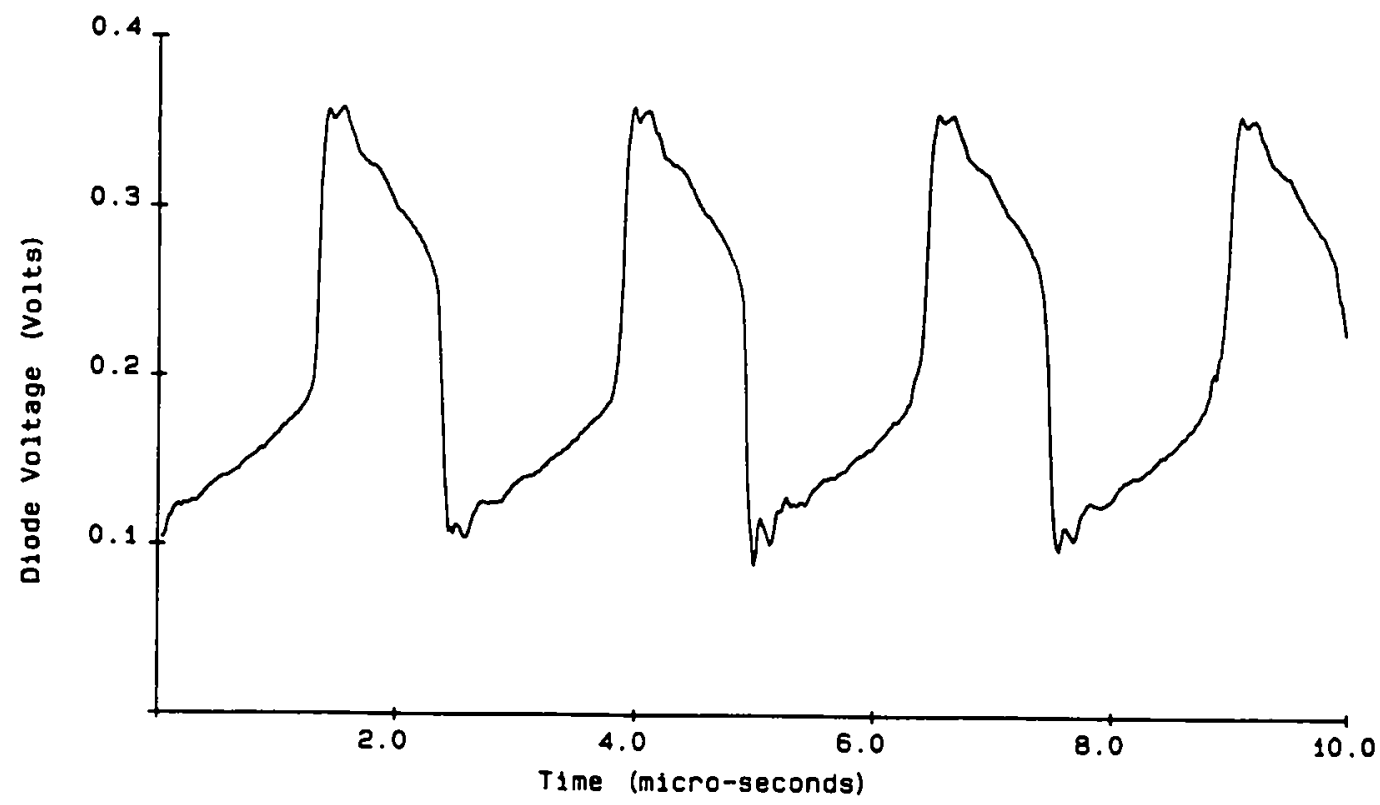

(b)

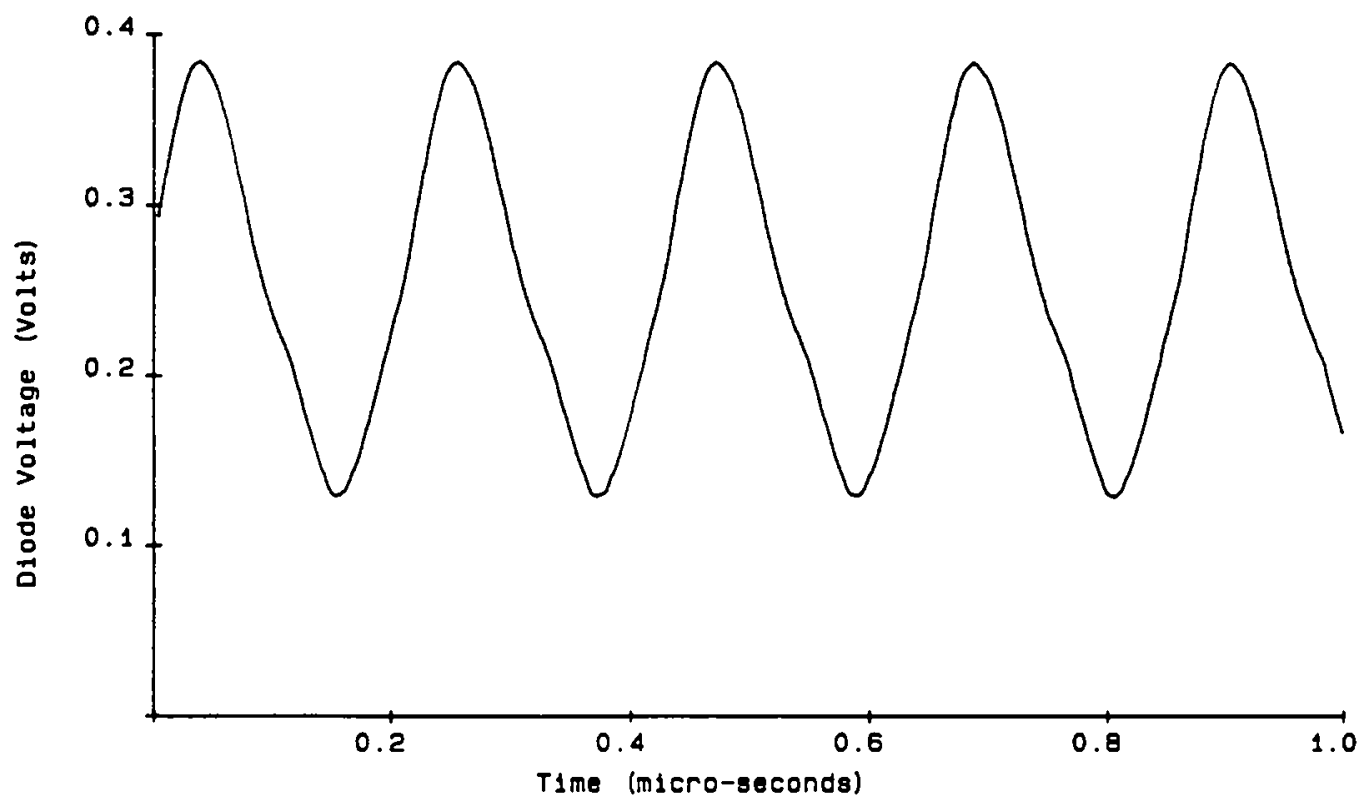

Fig. 3. Low frequency oscillation of the RTD as measured by an oscilloscope (a) in the growing exponential region (Area 1$)$ of the stability diagram. A large r.f. choke $\left(L_{\mathrm{s}} \approx 100 \mu \mathrm{H}\right)$ was placed in the bias lines to produce this oscillation, (b) in the growing sinusoidal region (Area II) of the stability diagram. The inductance is from approximately $2 \mathrm{~m}$ of lead wire $\left(L_{\mathrm{s}} \approx 2 \mu \mathrm{H}\right)$.

exponentially. The circuit is stable if it is in regions III or IV.

To obtain stability, the ratio $R_{\mathrm{s}} / R_{\mathrm{d}}$ should be just less than one so that both inequalities of eqn (1) can be satisfied. For a given device, $R_{\mathrm{d}}$ and $C_{\mathrm{d}}$ are fixed. Assuming the designer has control of $R_{\mathrm{s}}$ and $L_{\mathrm{s}}$ the stability criterion becomes

$$
L_{\mathrm{s}}<R_{\mathrm{d}}^{2} C_{\mathrm{d}}
$$

Typically, for RTDs with peak currents in the $\mathrm{mA}$ range $R_{\mathrm{d}}$ is tens of ohms or less and $C_{\mathrm{d}}$ is tens of $\mathrm{pF}$. This constrains $L_{\mathrm{s}}$ to $\mathrm{nHs}$ or even tenths of an $\mathrm{nH}$. Since a whisker contact introduces an inductance of this order, many RTDs cannot be stabilized when used with whisker contacts.

The stability diagram in Fig. 2 can also be used to study the effect of the series inductance $L_{\mathbf{s}}$. For a given device and circuit, the external inductance in the bias circuit can be varied to sample different portions of the stability plot. Bias-circuit oscillations 


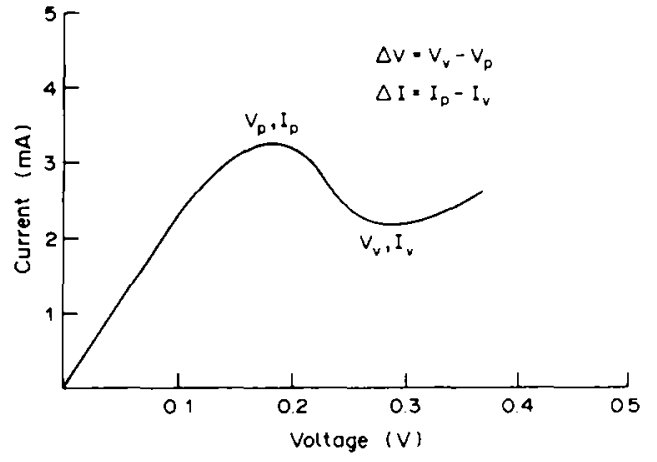

Fig. 4. The "true" stable (d.c.) / $V$ curve for the device under consideration as measured on the HP 4145 semiconductor parameter analyzer. Stabilization was attained using $R_{\mathrm{s}}=33 \Omega$ and $C_{\mathrm{c}}=0.1 \mu \mathrm{F}$.

of a resonant tunneling diode mounted in a lowinductance whisker-contact cavity with a variable external lead inductance are shown in Fig. 3. The device is a resonant tunneling diode with AlAs barriers that are $30 \AA$, thick, an $\operatorname{In}_{010} \mathrm{Ga}_{0.90}$ As deep well that is $70 \AA$ wide. and spacer layers on each side that are $30 \AA$ wide. The cladding regions are doped $2 \times 10^{1 \times} \mathrm{cm}^{3}$. Standard photolithiography techniques were used to fabricate diodes of various sizes. The measurements were done on 50 by $50 \mu \mathrm{m}$ diodes. The stable $I-V$ curve of this device is shown in Fig. 4.

The circuit is in region I of Fig. 2 for very large $L_{3}$. Devices biased in region I should have a growing exponential waveform. The growth is limited by the extent of the negative resistance region. If $R_{\mathrm{s}}$ is less than but almost equal to $R_{\mathrm{d}}$ the total resistance of the circuit will be negative only for a small region about the bias point and so the voltage swing will be further constrained. The result is the well known relaxation oscillation. Similar conditions occur in a tunnel diode[4]. The voltage across the bias terminals of the experimental device is shown in Fig. 3(a). For smaller values of $L_{5}$, the operating point moves to the left on the stability curve in Fig. 2 into region II. The stability diagram predicts growing sinusoidal oscillations, which are also limited by the extent of the negative resistance region. This results in a nearly sinusoidal steady state oscillation with a measured case shown in Fig. 3(b). In the growing sinusoid region, decreasing $L_{s}$ causes the frequency of oscillation to increase. This is shown by differentiating the frequency of oscillation with respect to the series inductance to obtain

$$
\frac{\mathrm{d}\left(\omega^{2}\right)}{\mathrm{d} L_{\mathrm{s}}}=\frac{1}{4 C_{\mathrm{d}}^{2} L_{\mathrm{s}}^{3}}\left(2 L_{\mathrm{s}} C_{\mathrm{d}}\left(\frac{R_{\mathrm{s}}}{R_{\mathrm{d}}}-2\right)+2 R_{\mathrm{s}}^{2} C_{\mathrm{d}}^{2}\right)
$$

For large $L_{\mathrm{s}}$ this is negative since $R_{\mathrm{s}}: R_{\mathrm{c}}<1$. The extremum is then found by setting the above equation equal to zero and solving for $L_{\mathrm{s}}$. An extremum occurs when

$$
L_{\mathrm{s}}=\left[\left(R_{\mathrm{s}}^{2} C_{\mathrm{d}}\right)\left(2-\frac{R_{\mathrm{s}}}{R_{\mathrm{d}}}\right)\right]<R_{\mathrm{s}}^{2} C_{\mathrm{d}}<R_{\mathrm{s}} R_{\mathrm{d}} C_{\mathrm{d}}
$$

Since the denominator of the derivative is linearly decreasing with $L_{s}$ this is a maximum. This means that the maximum frequency occurs for values of $L_{\mathrm{s}}$ smaller than the value required for stability. Now, if one calculates the oscillation frequency when $L$, is just small enough to give stability one obtains

$$
\text { (i) }=\frac{1}{R_{\mathrm{d}} C_{\mathrm{d}}} \sqrt{\frac{R_{\mathrm{d}}}{R_{\mathrm{b}}}}-1=()_{\mathrm{r}} .
$$

This gives physical meaning to the stability conditions. For a given device $R_{\mathrm{d}}$ and $C_{\mathrm{c}}$ are fixed. If $R_{\mathrm{s}}$ is less than $R_{\mathrm{d}}$, the circuit $L$, controls the stability. The idea is to decrease $L$, which in turn increases the oscillation frequency until the oscillation frequency is above the resistive cutoff frequency of the device.

A common method for stabilizing a tunnel diode or RTD is to place a capacitor in shunt across the terminals of the device[5 7]. From the preceding analysis it is seen that instability would be overcome if the d.c. source could be inserted physically near the RTD, minimizing the inductance. Since a large shunt capacitor will appear to the bias circuit oscillations as a d.c. voltage source the capacitance. $C_{\mathrm{e}}$ shown in Fig. 1(a) effectively accomplishes this.

To study the effect of $\mathrm{C}_{e}$ the circuit is broken into separate high-and low-frequency equivalents, both of the form of Fig. l(b). For high frequencies $C_{c}$ is a short circuit so the combination of $L_{5}, R_{\mathrm{s}}, R_{\mathrm{d}}$ and $C_{\mathrm{d}}$ in Fig. 1(a) must satisfy the stability criteria, eqn (1). For the low frequency circuit the element values in Fig. 1(b) become $L_{\mathrm{B}} \rightarrow L_{\mathrm{s}}, R_{\mathrm{B}} \rightarrow R_{\mathrm{s}} . R_{\mathrm{d}}-R_{\mathrm{s}} \rightarrow R_{\mathrm{d}}$ and $C_{\mathrm{c}} \rightarrow C_{\mathrm{d}}$. This low-frequency equivalent $R_{\mathrm{d}}$ is different from that given by Hines[5]. The value given here is chosen to give the correct impedance at d.c. Plotting the impedance locus vs frequency for various element values indicates that this approximation is very good when the high-frequency circuit is stable. Then a sufficient condition for the circuit in Fig. 1(a) to be stable is

$$
\frac{I_{\mathrm{v}}}{R_{\mathrm{d}}^{2} C_{\mathrm{b}}}<\frac{R_{\mathrm{b}}}{R_{\mathrm{c}}}<1
$$

and

$$
\frac{L_{\mathrm{B}}}{\left(R_{\mathrm{d}}-R_{\mathrm{S}}\right)^{2} C_{\mathrm{c}}}<\frac{R_{\mathrm{B}}}{\left(R_{\mathrm{d}}-R_{\mathrm{s}}\right)}<1
$$

When the high-frequency circuit is unstable the approximation is not always accurate. Nyquist analysis of the full circuit for different element values indicate that while it is theoretically possible to obtain a stable circuit when the high-frequency circuit is unstable, stability requires precise element values and could never be attained in practice. If the simple stability conditions, eqn (1) are not met by the circuit shunted by the external capacitance stable operation of the circuit in Fig. 1(a) is virtually unobtainable. 
Stabilization criteria for an equivalent circuit model including the effect of quasibound-state lifetime

Recently, an equivalent circuit has been proposed by Brown et al.[8] which is different from the tunnel diode equivalent circuit. The proposed circuit includes a negative inductance in series with the device negative resistance. The device intrinsic inductance is based on the idea that the time required for the buildup or decay of the wave function in the quantum well leads to a delay of current with respect to voltage. Using this model the authors have shown a better fit to the experimentally obtained power vs frequency data. This subsection describe the effect of this inductance on the stability criteria.

The differential equation representing the current $i$ through a short circuited device can be written as

$$
\begin{aligned}
& -L_{\mathrm{d}} L_{\mathrm{s}} C_{\mathrm{d}} \frac{\mathrm{d}^{3} i}{\mathrm{~d} t^{3}}-\left(R_{\mathrm{d}} C_{\mathrm{d}} L_{\mathrm{s}}+R_{\mathrm{s}} C_{\mathrm{d}} L_{\mathrm{d}}\right) \frac{\mathrm{d}^{2} i}{\mathrm{~d}^{2}} \\
& -\left(L_{\mathrm{d}}-L_{\mathrm{s}}+R_{\mathrm{s}} R_{\mathrm{d}} C_{\mathrm{d}}\right) \frac{\mathrm{d} i}{\mathrm{~d} t}-\left(R_{\mathrm{d}}-R_{\mathrm{s}}\right) \quad i=0
\end{aligned}
$$

Determining the stability using the Routh criterion one obtains the following sufficient condition

$$
\begin{aligned}
\left(R_{\mathrm{s}} L_{\mathrm{d}}+R_{\mathrm{d}} L_{\mathrm{s}}\right)\left(L_{\mathrm{d}}-L_{\mathrm{s}}+R_{\mathrm{s}}\right. & \left.R_{\mathrm{d}} C_{\mathrm{d}}\right) \\
& -L_{\mathrm{d}} L_{\mathrm{s}}\left(R_{\mathrm{d}} / R_{\mathrm{s}}\right)>0
\end{aligned}
$$

Simplifying this one obtains

$$
\frac{L_{\mathrm{s}}}{R_{\mathrm{d}}^{2} C_{\mathrm{d}}}<\frac{R_{\mathrm{s}}}{2 R_{\mathrm{d}}}\left(1+\sqrt{1+\frac{4 L_{\mathrm{d}}^{2}}{R_{\mathrm{d}}^{3} R_{\mathrm{s}} C_{\mathrm{d}}^{2}}+\frac{4 L_{\mathrm{d}}}{R_{\mathrm{d}}^{2} C_{\mathrm{d}}}}\right)
$$

The right hand side of eqn (9) is greater than $R_{\mathrm{s}} / R_{\mathrm{d}}$. Thus, the effect of the internal negative inductance is to relax the constraint on $L_{s}$. Note that it is shown that the device can be stable if the inductance is negative. If the internal inductance is assumed positive then the device will always be unstable. Since RTDs have been stabilized, if the proposed model is correct one needs to physically explain the existence and behaviour of a negative inductance.

\section{LOW FREQLENCY I-V CHARACTERISTICS OF UNSTABLE DEVICES}

The effect of various intrinsic and extrinsic circuit elements on the measured d.c. $I-V$ characteristics of unstable devices will be discussed in this section. It is shown that from the shape of the $I-V$ characteristic one can tell what kind of instability is present in the circuit. This analysis is not only useful for its own sake but it is necessary since the instability can have severe consequences on the device applications. Moreover, this also sheds some light on an ongoing debate among researchers on the nature of bistability in the device $[9,10]$. Some preliminary work on this subject has been described by Young et al.[7] and Liu[6]. The circuit of Fig. 1(b) will be used for the discussion of d.c. $I-V$ curves in this section. The diode used to produce the oscillations of Fig. 3 is used to experimentally demonstrate the conclusions,

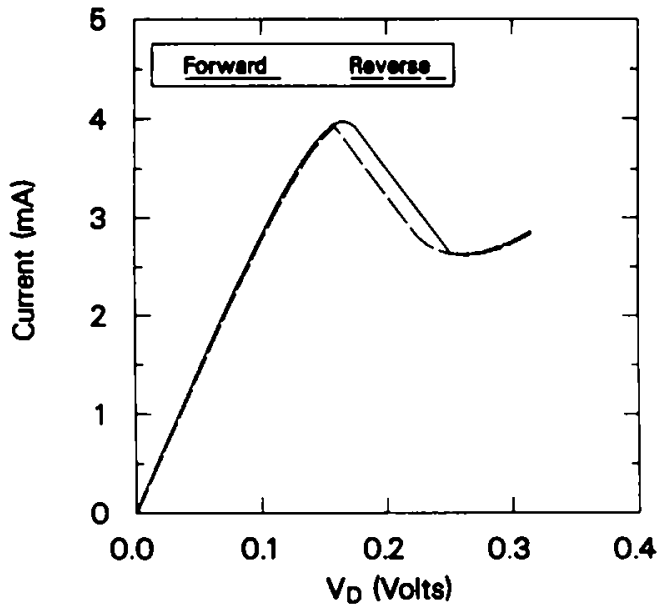

Fig. 5. The measured diode $I-V$ curve with an "external" resistance, $R_{\mathrm{s}} \approx 55 \Omega$. The "switching" of the diode is obvious. This type of a curve, with both the current and voltage discontinuous, would be expected from a curve tracer as used here.

except Fig. 10 where two of the diodes were contacted in parallel. The circuit of Fig. 1(a) was used to stabilize the diode with $R_{\mathrm{s}}=33 \Omega$ and $C_{\mathrm{e}}=0.1 \mu \mathrm{F}$. The diode was considered stable when no oscillations could be detected using an oscilloscope across the bias leads. Care was taken to ensure that the frequency of the oscillations, usually hundreds of $\mathrm{MHz}$, was not greater than the bandwidth of the oscilloscope. The stable $I-V$ curve of Fig. 4 is felt to represent the "true" $I-V$ curve. All the $I-V$ characteristics presented in this section except Fig. 7 were obtained experimentally using a semiconductor parameter analyzer.

Bias oscillations distort the measured $I-V$ characteristic away from the "ideal" curve of Fig. 4. Three classes of distortions are commonly observed:

1. Switching.

2. Bistability.

3. Bias circuit oscillations.

Sometimes more complex distortions such as double plateau structures are observed which have not been investigated.

An $I-V$ curve displaying switching is shown in Fig. 5. This type of curve is common when using an old style curve tracer, such as the Tektronix Type 575 Transistor-Curve Tracer. This type of distortion is discontinuous in both current and voltage. This behavior is due to a large series resistance between the voltage source and the point at which the voltage is measured. In Fig. 6 this corresponds to measuring $I$ vs $V_{D}$ with $R_{\mathrm{s}}>\left|R_{\mathrm{s}}\right|$ in the NDR region. The resulting $I-V$ curve is apparent from load line analysis on the stable $I-V$ characteristic shown in Fig. 4. As $V_{\text {in }}$ is increased from zero to $V_{\mathrm{p}}+R_{\mathrm{s}} I_{\mathrm{p}}$ the measured $I-V$ curve faithfully reproduces the true $I-V$ curve. Since no negative resistance is present the circuit is stable and there is no switching in the region 


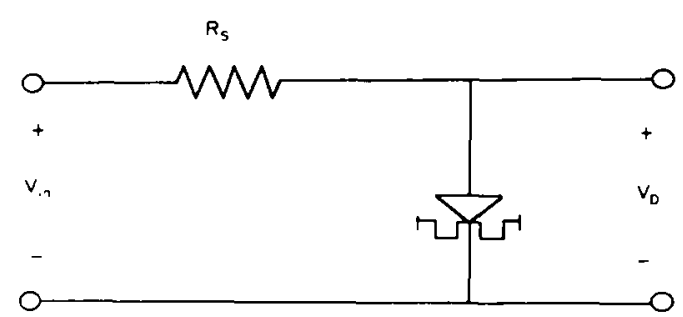

Fig. 6. The measurement circuit used to understand the effect of an external or internal series resistance.

$V_{\mathrm{v}}+R_{\mathrm{s}} I_{\mathrm{v}}<V_{\mathrm{in}}<V_{\mathrm{p}}+R_{\mathrm{s}} I_{\mathrm{p}}$ where the load line intersects the $I$ - $V$ curve at three different points. When $V_{\text {m }}$ is increased beyond $V_{\mathrm{p}}+R_{\mathrm{s}} I_{\mathrm{p}}$ the only stable point is on the right positive resistance portion of the $I-V$ curve, forcing switching behavior. When $V_{\text {in }}$ is swept from $V_{\text {in }}>V_{\mathrm{p}}+R_{\mathrm{s}} I_{\mathrm{p}}$ the same argument holds. So the $I-V$ curve for $V_{\mathrm{D}}>V_{v}$ is faithfully reproduced when the voltage is swept in the negative direction. Hysterisis occurs because the positive going switch point, $V_{\mathrm{tn}}=V_{\mathrm{p}}+R_{\mathrm{s}} I_{\mathrm{p}}$ is less than the negative going switch point. $V_{\text {In }}=V_{\mathrm{v}}+R_{\mathrm{s}} I_{\mathrm{v}}$. The switching is not instantaneous. The device capacitance does not allow the device voltage to change abruptly. The series inductance does not allow a discontinuous change in the current. Thus the device must spend some time in its NDR region. Since $R_{\mathrm{s}}>\left|R_{\mathrm{d}}\right|$ it follows from the stability diagram, Fig. 2, that the voltage is varying exponentially with time. It should be noted that the same chip was used for these measurements. The minor discrepancy between the peak currents in Figs 4 and 5 is thought to be due to the nonuniformity of the material.

Bistability in RTDs was first reported by Goldman et al.[9]. An $l-V$ curve showing bistability in the present device is shown in Fig. 7. This behavior is due to a voltage drop between the point at which the voltage is measured and the NDR device. In the inset of Fig. 6 this corresponds to measuring $I$ vs $V_{\text {in }}$ with $R,>\left|R_{\mathrm{d}}\right|$. For purposes of demonstration a large

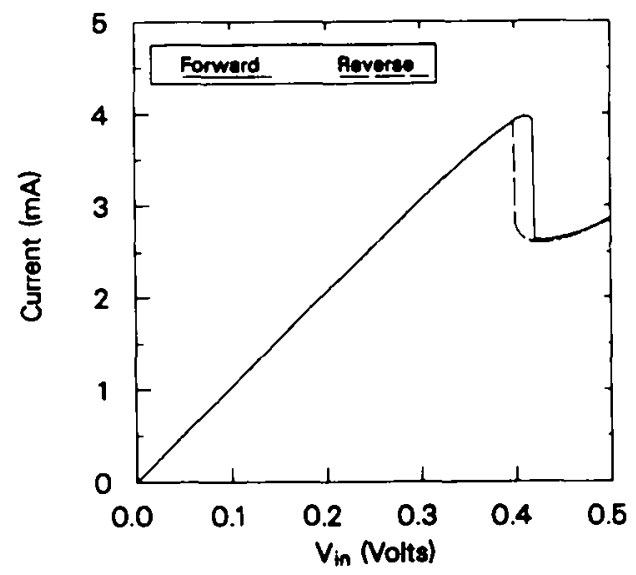

Fig. 7. Diode measured $I-V$ curve showing the bistable nature of the device due to an "intrinsic" series resistance. $R_{\mathrm{s}} \approx 55 \Omega$. The measurement circuit is shown in the inset. series resistance was added which was treated as "internal." In practice the internal series resistance is usually the positive resistance of the device independent of the measuring apparatus. This resistance includes contact resistance and epilayer resistance. The distinctive feature of this distortion is that only the current is discontinuous. Unlike the case of an external resistor, an internal resistance always distorts the $I-V$ curve, even when $R_{\mathrm{s}}<\left|R_{\mathrm{d}}\right|$ and there are no discontinuities. The quiescent diode voltage, $V_{\mathrm{D}}$ is related to the measured voltage, $V_{\text {in }}$ (see Fig. 6) by

$$
V_{\mathrm{in}}=V_{\mathrm{D}}+I R_{\mathrm{s}}
$$

The effect of the series resistance is to shift the $I-V$ curve to the right as shown in Fig. 8. Taking the derivative of eqn (10) gives

$$
\frac{\mathrm{d} V_{\text {in }}}{\mathrm{d} V_{\mathrm{D}}}=1+R_{\mathrm{s}} \frac{\mathrm{d} I}{\mathrm{~d} V_{\mathrm{D}}}
$$

When $\mathrm{d} V_{\text {in }} / \mathrm{d} V_{\mathrm{D}}<0$ the locus of $\left(I, V_{\text {In }}\right)$ curves back to the left and $I$ is no longer a single valued function of $V_{1 \mathrm{n}}$. This occurs in the NDR region when $R_{\mathrm{s}}>\left|R_{\mathrm{d}}\right|$ which is the exponentially increasing region of the stability diagram. This means points for which $\mathrm{d} V_{\text {in }} / \mathrm{d} V_{\mathrm{D}}<0$ cannot be stably biased and switching occurs if the external circuit attempts to sweep through these points. The physics behind the behavior is the same as in the switching case. The difference in the measured curves is due to measuring the voltage at different points in the circuit. In the bistable case there is no resistance between the voltage source and $V_{\text {in }}$ so the measured voltage will be continuous while the current is discontinuous. The voltage drop external to the well need not be due to a simple resistor. Similar effects may occur due to the accumulation and depletion layers within the device itself leading to the theoretical and experimental observation of what is now refered to as intrinsic bistability[11]. If the circuit is bistable much of the negative resistance region will be inaccessible to the external d.c. circuitry. It may be that if the r.f. frequency is high enough that the device is unable to switch during an r.f. cycle then the r.f. circuit will be able to take advantage of the negative resistance. This has not yet been clearly demonstrated. Even if this is the case the d.c. bias point will be severely restricted. Thus, for nonswitching applications bistability is very detrimental

An $I-V$ curve displaying a plateau structure is shown in Fig. 9. It is a simple matter to show by numerical methods that such a structure is to be expected when bias circuit oscillations are present[6]. The measured current is simply the time average of the current waveform. It does not involve a detection process, so the term "self detection" is a misnomer. These oscillations occur when

$$
\frac{R_{\mathrm{s}}}{R_{\mathrm{d}}}<\frac{L_{\mathrm{s}}}{R_{\mathrm{d}}^{2} C_{\mathrm{d}}} \quad \text { and } \quad \frac{R_{\mathrm{s}}}{R_{\mathrm{d}}}<1 .
$$




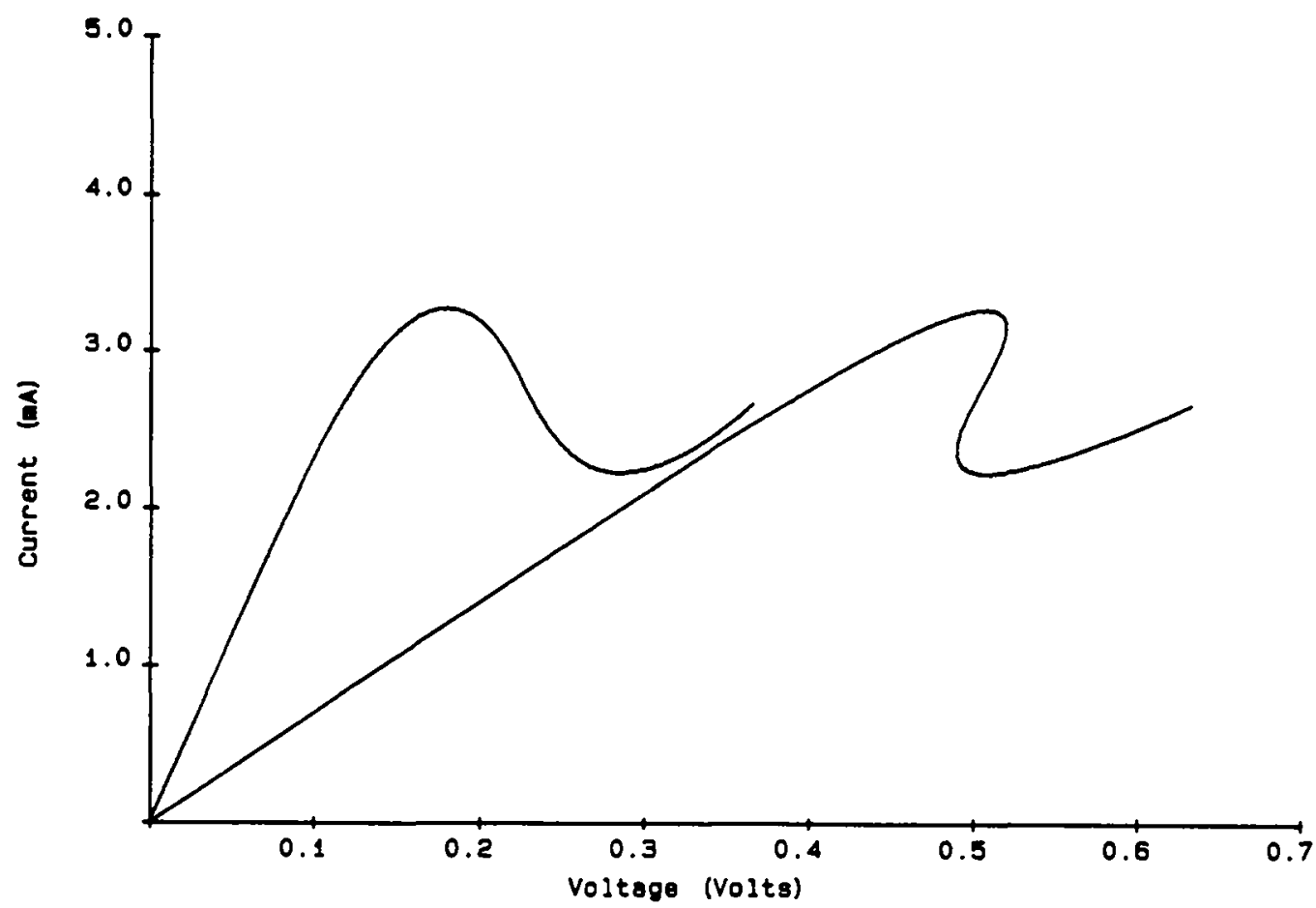

Fig. 8. Effect of an extrinsic series resistor on the measured $I-V$. "Shifted" curve is theoretically produced from the "true" $I-V$ curve. Internal resistance is $100 \Omega$.

In other words, when the device is in the unstable region of the stability diagram shown in Fig. 2. For the device tested the d.c. $I-V$ curve was insensitive to whether the oscillation was sinusoidal or relaxation, Figs 3(a) or (b). It is not understood at this point why the d.c. curve is insensitive to sinusoidal vs relaxation oscillations.

The analysis of the effect of a series resistor on the $I-V$ curve of an oscillating device follows the same procedure as for a stable device. The case of an internal series resistance will be discussed in detail. For this discussion it is assumed that the circuit oscillates whenever the RTD has a negative differen-

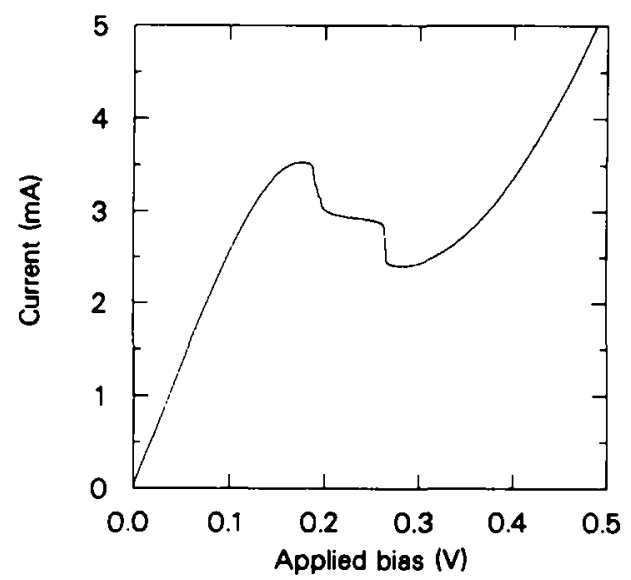

Fig. 9. I-V curve showing the effect of circuit oscillations that results in the plateau like structure. No series resistance was intentionally introduced. tial resistance. The d.c. current is approximated as constant at $I_{\text {plat }}$ for $V_{\mathrm{p}}<V_{\mathrm{D}}<V_{\mathrm{v}}$. As long as the circuit is in a stable mode the circuit will not change modes when the voltage is perturbed slightly or swept slowly. Starting at zero volts and sweeping in the positive direction, no oscillations occur until $V_{\mathrm{D}}$ is equal to $V_{\mathrm{p}}$ which corresponds to $V_{\text {in }}=V_{\mathrm{p}}+I_{\mathrm{p}} R_{\mathrm{s}}$. When $V_{\text {in }}$ is increased beyond this point the circuit starts oscillating and the current switches to $I_{\text {plat }}$. As the input voltage is increased further the diode continues to oscillate until $V_{\mathrm{D}}$ exceeds $V_{\mathrm{v}}\left(V_{\text {in }}=V_{\mathrm{v}}+I_{\text {plat }} R_{\mathrm{s}}\right)$. For larger $V_{\text {in }}, V_{\mathrm{D}}>V_{\mathrm{v}}$ and the oscillation is quenched and the current changes from $I_{\text {plat }}$ to the value on the right hand positive resistance region determined by the load line analysis. Starting at a large $V_{\text {in }}$ and sweeping in the negative direction, no oscillations occur until $V_{\mathrm{D}}$ is equal to $V_{v}$ which corresponds to $V_{\text {in }}=V_{\mathrm{v}}+I_{\mathrm{v}} R_{\mathrm{s}}$. When $V_{\text {in }}$ is decreased beyond this point the circuit starts oscillating and the current switches to $I_{\text {plat }}$. The circuit continues to oscillate as the voltage is swept until $V_{\mathrm{D}}$ is less than $V_{\mathrm{p}}\left(V_{\text {in }}=V_{\mathrm{p}}+I_{\mathrm{plat}} R_{\mathrm{s}}\right)$. When $V_{\text {in }}$ is decreased beyond this point the oscillation is quenched and the current jumps from $I_{\text {plat }}$ to the value of the left hand positive resistance region determined by the load line analysis. Since $V_{\mathrm{p}}+I_{\mathrm{p}} R_{\mathrm{s}}>V_{\mathrm{p}}+I_{\mathrm{plat}} R_{\mathrm{s}}$ the $I-V$ curve is bistable at the lower boundary of oscillation. Since $V_{\mathrm{v}}+I_{\mathrm{plat}} R_{\mathrm{s}}>V_{\mathrm{v}}+I_{\mathrm{v}} R_{\mathrm{s}}$ the $I-V$ curve is bistable at the upper boundary of oscillation. In contrast to the stable diode case an internal resistor always causes bistability in an oscillating case. An experimental example of bistability in an oscillating circuit is 


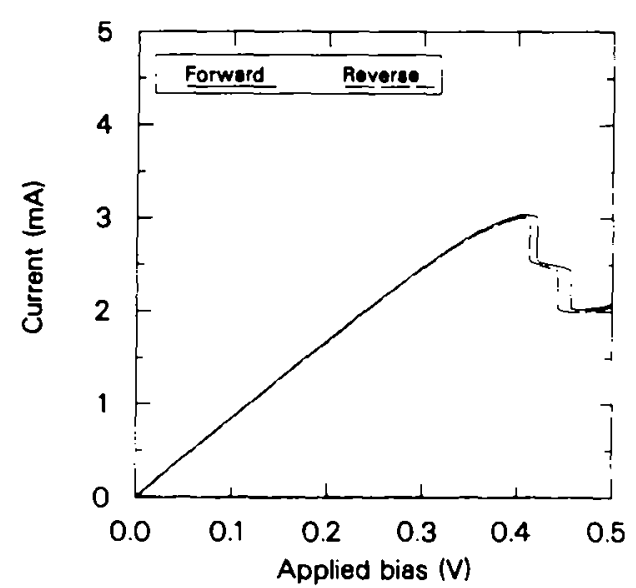

Fig. 10. $I V$ curve for a device that has an internal resistance and is also oscillating. $R_{s} \approx 33 \Omega$.

shown in Fig. 10, A similar argument shows that switching behavior occurs whenever there is an external resistor in series with an oscillating device. Figure 11 shows an experimental example of this switching.

\section{CONCI.USION}

The stability criteria for resonant tunneling diodes have been derived. Based on the criteria it is shown that stable operation of resonant tunneling diodes is hard to obtain. The effect of extrinsic and intrinsic elements on the criteria for stability has been discussed including using an external capacitance to stabilize the negative resistance device. The importance of circuit inductance cannot be over emphasized. In order to stably bias an RTD the lead inductance must be minimized. The diodes can be made stable by using a shunt capacitor but this is only possible if the circuit inductance is very small. It was shown theoretically that each instability produces a signature $I-V$ characteristic. The expected $I-V$ curves were experimentally produced using a diode which could be stabilized. It was shown that bistability of a device does not imply bistability of the double barrier structure itself and that the question of whether bistability is intrinsic or extrinsic is largely a question of semantics.

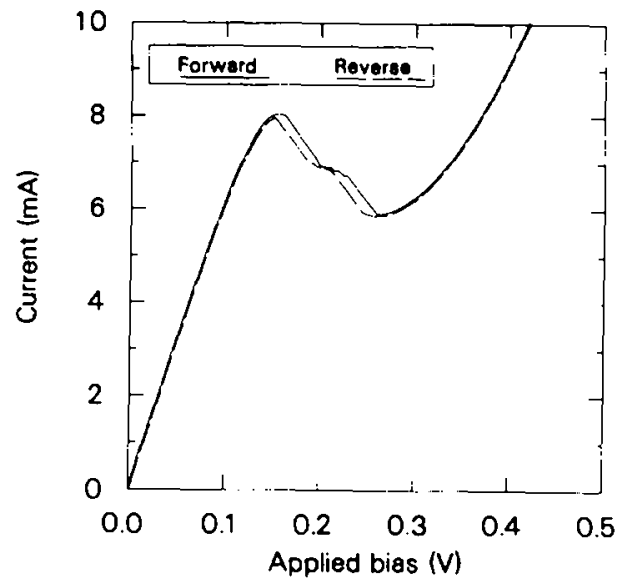

Fig. 11. I-V curve for a device that has an external resistance and is also oscillating. $R_{\mathrm{s}} \approx 55 \Omega$ (device area in this case is 100 by $100 \mu \mathrm{m})$.

Acknowledgements - The authors wish to thank Dr Richard Mains for many helpful discussions. Special thanks to Ms Traci Jones for help with the manuscript. This work was supported by the NASA Center for Space Terahertz Technology under contract no. NAGW-1334 and the U.S. Army Research Office under the URI program, contract no. DAAL03-87-K-007.

\section{REFERENCES}

1. E. R. Brown, T. C. L. G. Sollner, C. D. Parker. W. D Goodhue and C. L. Chen, Appl. Phys. Lell. 55, 1777 (1989).

2. C. Kidner, I. Mehdi, J. East and G. Haddad, IEEE Trans. Microwave Theory Technol. 38, 864 (1990).

3. H. A. Watson, Microwate Semiconductor Devices and their Circuit Applications. McGraw-Hill, New York (1969).

4. W. F. Chow, Principles of Tunnel Diode Circuits. Wiley. New York (1964).

5. M. E. Hines, Bell Syst. tech. J. 39, 477 (1960)

6. H. C. Liu, Appl. Phys. Lett. 53, 485 (1988).

7. J. F. Young, B. M. Wood, H. C. Liu, M. Buchanan, D. Landheer, A. J. SpringThorpe and P. Mandeville. Appl. Phys. Lett. 52, 398 (1988).

8. E. R. Brown, C. D. Parker and T. C. L. G. Sollner. Appl. Phys. Lell. 54, 934 (1989).

9. V. J. Goldman, D. C. Tsui and J. E. Cunningham, Phys. Rev. Lett. 58, 1256 (1987).

10. T. C. L. G. Sollner, Phys. Ret, Lett. 59, 1622 (1987).

11. R. K. Mains. J. P. Sun and G. I. Haddad, Appl. Phy's. Lett. 55, 371 (1989). 